\title{
Protéase 26S de levure, cycle cellulaire et oncogenèse
}

Chez les eucaryotes, la dégradation des protéines à vie brève commence généralement par la fixation covalente d'un polypeptide, l'ubiquitine, sur la protéine à dégrader. Les protéines ubiquitinylées peuvent être alors dégradées par un complexe macromoléculaire, la protéase $26 \mathrm{~S}$. Celle-ci est formée par l'association du protéasome 20S, une protéase aux propriétés multicatalytiques, avec un ensemble de 10-20 sousunités supplémentaires. Cette association confère à l'ensemble la capacité de reconnaître et de dégrader d'une façon dépendante de l'ATP les protéines multi-ubiquitinylées [1]. La caractérisation moléculaire des sous-unités spécifiques à la protéase $26 \mathrm{~S}$ en est à ses tout débuts. Trois laboratoires viennent de publier dans le même numéro de Nature une série d'observations complémentaires sur l'identité de certaines de ces sous-unités et leur rôle dans le cycle cellulaire et l'oncogenèse. Ces informations ont toutes été obtenues par la mise en œuvre d'outils de la génétique moléculaire des levures.

Ghislain et al. (service de biochimie et de génétique moléculaire, CEASaclay) ont isolé deux mutants thermosensibles de Saccharomyces cerevisiae, cim3-1 et cim5-1, qui ont la propriété de stopper leur division en phase G2 du cycle cellulaire avec une configuration métaphasique du fuseau mitotique [2]. Donc, l'ADN génomique est dupliqué normalement chez les mutants, mais la lieu. Les protéines CIM3 et CIM5 sont à $40 \%$ identiques en acides aminés et elles appartiennent toutes les deux à la famille des ATPases. En outre, la protéine humaine MSSI est à $70 \%$ identique à CIM5 et MSS1 peut fonctionnellement remplacer CIM5 chez la levure. Selon les auteurs, CIM3 et CIM5 pourraient être deux des sous-unités qui s'assemblent au protéasome $20 \mathrm{~S}$ pour former la protéase 26S. Cette hypothèse est confortée par le fait qu'ils retrouvent des homologues de CIM3 et CIM5 dans une préparation quasi homogène de la protéase $26 \mathrm{~S}$ de drosophile. De plus, une protéine de fusion ubiquitinylée (ubiquitine-proline-- $\beta$-galactosidase), normalement dégradée chez la levure sauvage, est stabilisée chez les mutants cim. Cependant, une classe de protéines contenant un acide aminé amino-terminal déstabilisant, également dégradée par une voie dépendante de l'ubiquitine, n'est pas stabilisée chez les mutants cim. Les protéines CIM3 et CIM5 seraient donc nécessaires à la reconnaissance et au clivage d'une classe de protéines ubiquitinées, alors que d'autres sous-unités à activité ATPase, pas encore identifiées, seraient indispensables pour accomplir ces fonctions vis-à-vis d'autres familles de protéines ubiquitinylées. Gordon, et al. (MRC, Edinburgh, GB) ont identifié dans la protéase $26 \mathrm{~S}$ de la levure Schizosaccharomyces pombe une autre sousunité à fonction ATPasique [3], dont l'inactivation conduit à un arrêt métaphasique ressemblant à celui des mutants cim de Saccharomyces cerevisiae. Ainsi, chez les deux levures, la protéase $26 \mathrm{~S}$ est nécessaire pour passer de métaphase en anaphase au cours de la mitose. Une étude in vitro avec des extraits d'ovocyte de xénope est arrivée à la même conclusion [4].

Quelles sont les protéines qui doivent être dégradées pour permettre le passage en anaphase et la séparation chromosomique? On sait que l'activité kinasique MPF (mitosis promoting factor), composée d'une sousunité catalytique CDC2/CDC28 en association avec une sous-unité activatrice appelée cycline, déclenche l'entrée en mitose. Les cyclines mitotiques sont spécifiquement protéolysées pendant la mitose, et leur dégradation entraîne l'inactivation de la kinase CDC2/CDC28 et la sortie de la mitose. Des études récentes indiquent que la stabilisation d'une seule cycline n'empêche pas la ségrégation chromosomique [4, 5], mais il n'est pas exclu que la dégradation de l'ensemble des cyclines mitotiques (chaque cellule eucaryote en exprime plusieurs) soit nécessaire pour l'entrée en anaphase. Selon un autre modèle, une protéine hypothétique liant les chromatides sœurs pourrait être la cible de dégradation [4].

Un autre éclairage sur le rôle de la protéase 26S est apporté par le travail de Papa et Hochstrasser (université de Chicago, USA) qui décrivent l'isolement d'un mutant de Saccharomyces cerevisiae, doa4, dans lequel la demi-vie de la protéine instable 
Mat $\alpha 2$ est augmentée. La protéine DOA4 s'avère être un nouveau type de dé-ubiquitinase qui a une séquence similaire au produit de l'oncogène tre-2[6]. Les déubiquitinases sont une famille d'enzymes catalysant le clivage de l'ubiquitine des protéines multiubiquitinylées. L'analyse biochimique du mutant suggère que DOA4 serait spécifiquement chargée de la dé-ubiquitinylation des protéines en cours de dégradation par la protéase 26S. En son absence, l'accumulation à l'intérieur de la protéase 26S (un complexe macromoléculaire en forme de cylindre) de peptides ubiquitinylés bloquerait les sites actifs de la protéase, de telle sorte que d'autres substrats ne pourraient y avoir accès. La forme oncogénique de la protéine Tre-2 serait une forme tronquée de cette dernière, sans activité catalytique, mais capable d'empoisonner la fonction de la protéase 26S. Dans ces conditions, les produits d'autres protooncogènes, qui sont souvent des protéines à vie brève, pourraient s'accumuler et stimuler la division cellulaire.

C.R.

1. Rechsteiner M, Hoffman 1, Dubiel W. The multicatalytic and $26 \mathrm{~S}$ proteases. J Biol Chem 1993 ; $268: 6065-8$.

2. Ghislain M, Udvardy A, Mann C. S. cerevisiae $26 \mathrm{~S}$ protease mutants arrest cell division in G2/metaphase. Nature 1993; 366: 358-62. 3. Gordon C, McGurk G, Dillon P, Rosen C, Hasues ND. Defective mitosis due to a mutation in the gene for a fission yeast $26 \mathrm{~S}$ protease subunit. Nature 1993; 366: 355-7. 4. Holloway SI, Glotzer M, King RW, Murray AW. Anaphase is initiated by proteolysis rather than by the inactivation of maturationpromoting factor. Cell 1993; 73 : 1393-402. 5. Surana U, Amon A, Dowzer C; McGrew J, Byers B, Nasmyth K. Destruction of the C.DC:28/C.1.B mitotic kinase is not required for the metaphase to anaphase transition in budding yeast. EMBO J 1993; 12 : 1969-78. 6. Papa FR, Hochstrasser M. The yeast $D() A 4$ gene encodes a deubiquitinating enzyme related to a product of the human tre-2 oncogene. Nature 1993; 366: 313-9.

$\mathrm{m} / \mathrm{s} n^{\circ} 2$ vol. 10, färier 94 n'entraîne pas de réponse anticoagulante, ce qui a été interprété comme le manque d'un co-facteur nécessaire à l'activité de la protéine C. La récente étude castémoin de Koster et al. de l'université de Leyden (Pays-Bas) [3], effectuée chez 301 malades présentant pour la première fois une thrombose des veines profondes, montre que la fréquence de cette anomalie $(21 \%)$ est plus élevée que les déficits congénitaux en protéine $\mathrm{C}$, protéine $S$ ou anti-thrombine III $(<10 \%)$ [4]. Il s'agit d'une nouvelle anomalie dans le système de coagulation, inconnue jusqu'à présent. L'anomalie per se n'est pas suffisante pour produire une thrombose, ainsi qu'en témoigne sa fréquence $(5 \%)$ dans la population contrôle. Il ne s'agirait donc pas d'une affection monogénique ; d'autres facteurs sont nécessaires pour que cette anomalie puisse exprimer son potentiel thrombo- gène, facteurs acquis ou facteurs héréditaires encore inconnus. Cela est également vrai pour les déficits congénitaux en protéine C. Quand ces éléments sont présents, une faible réponse anticoagulante à la protéine $\mathrm{C}$ activée favorise de façon importante le développement des thromboses. Le défaut qui soustend cette condition pathologique est encore inconnu; il s'agit vraisemblablement du déficit d'un cofacteur de la protéine C activée, transmis de façon autosomique dominante; la faible réponse à la protéine $\mathrm{C}$ activée est cinq à dix fois plus fréquente que les déficits en protéine $C$, protéine $S$ et antithrombine III, et confère le même risque de thrombose veineuse [3]. Il semble donc important aujourd'hui de rechercher cette anomalie chez tous les malades qui thrombosent.

[1. Dahlbäck B, et al. Proc Natl Acad Sci USA 1993; 90: 1004-8.]

[2. Svensson PJ, Dahlbäck B. Thomb Haemost 1993; 69 : 2534 (Abstr).] [3. Koster $\mathrm{T}$, et al. Lancet 1993; 342: 1503-6.]

[4. Miletich JP, et al. Cell 1993; 72: 477-80.]
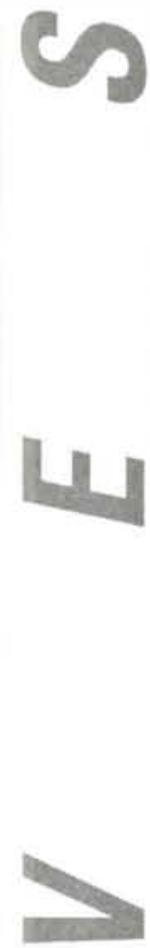

Interféron $\gamma$ et tuberculose. In vitro, l'interféron $\gamma$ (IFN $\gamma$ ) inhibe la croissance des mycobactéries mais son rôle protecteur in vivo n'avait jamais été clairement démontré. L'infection par Mycobacterium tuberculosis, de souris IFN $\gamma-/-$, récemment obtenues par knock-out [1], permet aujourd'hui d'affirmer que l'IFN $\gamma$ est aussi indispensable, in vivo, au développement d'une immunité cellulaire protectrice. T. Stewart et D. Dalton (Genentech Inc, USA), producteurs des souris IFN $\gamma-/-$, associés à deux groupes de laboratoires travaillant apparemment en concurrence, publient deux articles partiellement complémentaires, ou redondants, selon le caractère positif ou négatif 
que l'on attribue à la multiplication de publications si proches, en particulier lorsqu'elles émanent en partie des mêmes auteurs [2, 3]. Dans le premier [2], l'analyse histologique des lésions qui surviennent chez les animaux déficitaires IFN $\gamma-/-$ infectés par voie intraveineuse ou par aérosol révèle une nécrose caséeuse disséminée dans les poumons et le foie et l'absence de réponse cellulaire à l'acmé de la bactériémie alors qu'une réponse granulomateuse est observée au début de l'infection. Il semble donc que la formation de granulome soit sous la dépendance d'autres cytokines que l'IFN $\gamma$. Dans la seconde publication, il est démontré que l'apport de IFN $\gamma$ exogène permet de retarder l'évolution mais ne peut empêcher la survenue de la mort des animaux infectés, et que l'IFN $\gamma$ agit via la production d'intermédiaires réactionnels de l'azote par la NO synthétase des macrophages dont l'expression se trouve induite dans ces conditions. Ces résultats confirment le rôle prépondérant de l'immunité à médiation cellulaire dans la résistance à la tuberculose et suggèrent que le traitement par l'IFN $\gamma$ des tuberculoses qui surviennent en particulier chez les patients atteints de SIDA pourraient, non pas éliminer, mais au moins concourir à limiter la dissémination de Mycobacterium tuberculosis.

[1. Dalton DK, et al. Science 1993; 259: 1739-41.]

[2. Cooper AM, et al. / lixp Med 1993; 178: 2243-7.]

[3. Flynn JL, et al. J Exp Med 1993; 178: 2249-54.] spécifique (protéine découplante) qui n'est pas couplé aux phosphorylations oxydatives. De ce fait, l'ouverture de ce canal entraîne une dissipation du gradient de protons mitochondrial sans synthèse d'ATP, mais avec production de chaleur. L'activité de cette protéine découplante est stimulée par innervation sympathique et par les agonistes $\beta$-adrénergiques [1]. Outre son intervention évidente dans la thermogenèse et dans l'adaptation au froid, la graisse brune a depuis longtemps été soupçonnée de jouer également un rôle important dans le contrôle du poids corporel. En effet, une diminution de la dépense énergétique due à une défaillance quantitative ou qualitative de la graisse brune constitue un mécanisme très plausible de certaines formes d'obésité [1]. Une équipe américano-canadienne (Boston, MA, USA ; Ottawa, Canada ; Bar Harbor, Maine, USA) [2] vient de démontrer la réalité de cette hypothèse grâce à la technique de la toxi-génétique che\% des souris transgéniques. Des souris transgéniques ont été créées, chez lesquelles le gène codant pour la toxine diphtérique a été placé sous le contrôle des régions régulatrices du gène de la protéine découplante. L'expression de la toxine diphtérique dans le tissu adipeux brun provoque sa destruction, associée au développement d'une obésité qui s'accompagne, au bout de plusieurs mois, d'une résistance sévère à l'insuline. Tardivement, se développe même une hyperphagie. Auparavant, l'obésité se développe alors que les animaux ont une consommation normale de nourriture, ce qui est cohérent avec l'hypothèse que le mécanisme en est une diminution de la dépense énergétique due à la disparition du tissu adipeux brun. Cependant, on ne peut écarter complètement l'intervention d'autres mécanismes, notamment au niveau du système nerveux central où une expression très faible de la protéine découplante a été notée, de telle sorte que la destruc- tion de certaines populations neuronales ne peut être complètement éliminée chez les animaux transgéniques.

[1. Ricquier D. médecine/sciences 1985; 1: 147-53.]

[2. Lowell PB, et al. Nature 1993; 366 : 740-2.]

Interleukine 1, apoptose et sénescence. En octobre 1990, médecine/sciences rapportait les résultats remarquables de Maier et al concernant la prolongation de la vie de cellules endothéliales à l'aide d'un oligonucléotide antisens dirigé contre l'ARNm de l'interleukine-l $\alpha$ [1] $\left(\mathrm{m} / \mathrm{s}, n^{\circ} 8\right.$, vol. 6, p.811). ('est au rôle possible de l'interleukine-l dans les processus d'apoptose et de sénescence que ramène une recherche débutée chez le nématode Caenorhabditis elegans, par le laboratoire de HR Horvitz (MIT, Cambridge, MA, USA) [2]. J Yuan, et al. ont en effet cloné le gène ced-3, impliqué dans les phénomènes de mort programmée associés au développement de Caenorhabditis elegans. De la séquence de ce gène ced-3, les auteurs ont pu déduire qu'il codait pour une protéase à cystéine ressemblant étrangement à l'enzyme de mammifère ICE (interleukin-1- $\beta$ converting enzyme). En réalité ced 3 semble l'homologue, chez Caenorhabditis ele gans, du gène ICE chez les mammifères. Ia protéine ICE, comme son nom l'indique, assure la maturation protéolytique de la prointerleukine-1 $\beta$ de $33 \mathrm{kDa}$ en sa forme biologiquement active de $17,5 \mathrm{kDa}$. L'hyper-expression du gène $I C E$ ou du gène $c e d-3$ dans des cellules de rat en culture induit leur apoptose. Cette évolution est bloquée par l'hyper-expression du gène $b c l 2$ ou d'un gène du virus de la vaccine, $\mathrm{crm} A$. Ce gène viral est un inhibiteur de l'enzyme ICE qui intervient pour retarder la mort 
cellulaire en bloquant l'apoptose, facilitant ainsi la réplication virale [3]. Naturellement, il n'est pas sûr que, dans le cas du produit du gène $c e d-3$ et de l' $I C E$, l'action passe par l'intermédiaire d'une activation d'une interleukine-l qui serait responsable de l'effet apoptotique. Ces protéases peuvent, en effet, avoir d'autres cibles non encore déterminées et, d'autre part, l'expression des interleukines-l semble très faible dans la plupart des cellules. Il n'empêche que la coïncidence est pour le moins troublante et devrait inciter les auteurs à tester le rôle d'analogues de l'interleukine-l dans l'induction de l'apoptose de cellules diverses, chez les mammifères comme chez Caeno rhabditis elegans.

[1. Maier JAM, et al. Science 1990; 249 : 1570-4.]

[2. Yuan J, et al. Cell 1993; 75 : 641-52.]

[3. Miura L, et al. Cell 1993; 75 : 65.3-60.]

Le partenaire commun des récepteurs des interleukines 2,4 et 7. Les récepteurs des interleukines sont presque tous hétéromultimériques, certaines de leurs sous-unités étant communes à plusieurs récepteurs [1] $\left(\mathrm{m} / \mathrm{s}, n^{\circ} 5\right.$, vol. 8, p. 490). Cette tendance des récepteurs de cytokines à l'échange de partenaires explique le paradoxe qu'a fait naître il y a quelques mois la découverte du gène responsable du syndrome de déficience immunitaire combinée sévère liée à l'X chez l'homme. Ce gène est en effet la sous-unité $\gamma$ du récepteur de l'interleukine 2 [2]. Cependant, cette observation ne pouvait être expliquée simplement par une interruption du signal déclenché par l'IL2 puisque la mutation homozygote du gène $I L 2$, obtenue par recombinaison homologue chez les souris ou spontanée chez l'homme, n'est associée qu'à une déficience immunitaire très modérée $[3,4]$. Plusieurs équipes se sont donc efforcées de déterminer si la sous-unité $\gamma$ impliquée dans le déficit immunitaire lié à l'X chez l'homme n'était pas un constituant des récepteurs pour d'autres cytokines. Des chercheurs du NIH à Bethesda (MD, USA) [5, 6] de Sendaï et de Tokyo au Japon [7] viennent maintenant de démontrer que cette sous-unité $\gamma$ était également une composante des récepteurs de l'interleukine 4 et de l'interleukine 7. Les malades atteints du déficit immunitaire lié à l'X sont donc déficients dans la transmission des signaux issus de ces trois cytokines qui interviennent à différents niveaux du développement et de la différenciation des cellules lymphocytaires T et B. De plus, des résultats préliminaires font supposer qu'au moins un autre récepteur pourrait inclure cette sous-unité $\gamma$, celui de l'interleukine 13 [8].

[1. Wendling F. médecine/sciences $1991 ; 7$ : 569-77.]

[2. Noguchi M, et al. Cell 1993; 73 : 147-57.]

[3. Weinberg K, Parkman R, N ling J Med 1990; 322: 24 : 1718-23.]

[4. Schorle H, et al. Nature 1991; 352: 621-4.]

[5. Noguchi M, et al. Science 1993; 262 : 1877-80.]

[6. Russell SM, et al. Science 1993; 262: 1880-2.]

[7. Kondo M, et al. Science 1993; 262 : 1874-7.]

[8. Nowak R. Science 1993; 262 : 1818.]

Le précurseur des lymphocytes Thl et Th2 exprime le gène de l'interleukine 4. Les clones de lymphocytes T CD4 peuvent être regroupés en deux catégories principales sur la base des cytokines qu'ils sécrètent. Les lymphocytes Thl interviennent dans l'hyper- sensibilité retardée et l'activation des macrophages; ils sécrètent les cytokines pro-inflammatoires telles que l'interféron $\gamma$ (IFN $\gamma)$ et le facteur nécrosant des tumeurs. Les lymphocytes Th2, quant à eux, aident à la production d'anticorps par les cellules B et sécrètent l'IL4, l'IL5, l'ILl 0. Il est généralement admis que les cellules Thl et Th2 coexistent in vivo et que leur contribution respective à la défense de l'organisme varie en fonction du type d'antigènes ou de pathogènes rencontrés ([1] et $m / s n^{\circ} 5$, vol. 7, p. 515). L'acquisition du phénotype Thl ou Th2 par une cellule T CD4 native résulte de plusieurs événements de différenciation dont la séquence mérite d'être découverte pour de multiples raisons. Pour ne citer qu'un exemple, le rapport Thl/Th2 détermine la qualité de la réponse immunitaire, et l'utilisation excessive du phénotype Th2 peut conduire à des réactions allergiques. L'IL4, qui joue un rôle critique dans l'établissement d'une réponse de type Th2, semble être un des facteurs essentiels de la différenciation des cellules $\mathrm{T}$ natives. Pour préciser l'importance de l'IL4 dans la genèse des cellules effectrices Thl et Th2, des lignées de souris ont été créées chez lesquelles les cellules exprimant le gène IL4 peuvent être sélectivement éliminées [2]. Chez ces souris transgéniques, le promoteur de l'IL4 contrôle la synthèse de la thymidine kinase du virus Herpes simplex (HSV-TK) qui rend les cellules sensibles au ganciclovir $\left(m / s, n^{\circ} 7\right.$, vol. 8, p. 728). L'élimination des lymphocytes $\mathrm{T}$ transgéniques en présence de ganciclovir fait disparaître non seulement la production d'IL4 mais aussi celle de l'IFN $\gamma$. Cela montre clairement que la grande majorité des lymphocytes Thl (producteurs d'IFN $\gamma$ ) et des lymphocytes Th2 (producteurs d'IL4) proviennent de la différenciation d'un précurseur commun qui, lui aussi, exprime le gène de l'IL4. Ces souris ne sont pas seulement utiles pour examiner la mise en place du rapport (1) 
Thl/Th2 mais elles fournissent également un moyen d'étude in vivo des cellules productrices d'IL4. Il est maintenant possible de séparer, lors d'infections expérimentales, les effets des cellules exprimant le gène IL4 de ceux de l'IL4 elle-même.

[1. Capron A, Dessaint JP. médecine/sciences $1990 ; 6$ : 958-65.]

[2. Kamogawa Y, et al. Cell 1993; 75: 985-95.]

Le ligand de Fas est un membre de la famille du facteur nécrosant des tumeurs. L'antigène Fas est un membre de la famille des récepteurs du facteur de croissance des nerfs et du facteur nécrosant des tumeurs (TNF). Il est exprimé par différents tissus, dont le thymus, le foie, les poumons et le cour. Des anticorps monoclonaux anti-Fas induisent l'apoptose des cellule Fast, aussi bien in vivo qu'in vitro, suggérant que Fas transduit des signaux d'apoptose lorsqu'il s'associe à son ligand naturel ([1] $\left(m / s n^{\circ} 11\right.$, vol. 9, p. 1279)). En outre, la mutation homozygote du gène Fas chez la souris Ipr se traduit par une lymphadénopathie et des réactivités auto-immunes $(\mathrm{m} / \mathrm{s}$ $n^{\circ} 7$, vol. 8, p. 735). Chez ces souris, Fas n'est pas exprimé sur les thymocytes qui, de ce fait, échappent à la sélection thymique par apoptose. Fas n'est pas seulement impliqué dans le développement des lymphocytes $\mathrm{T}$ : l'activité cytotoxique de certaines cellules ne peut s'affirmer que si Fas est exprimé sur la cellule cible, probablement parce que les effecteurs sont porteurs du ligand. C'est en tout cas ce qu'a pensé l'équipe franco-japonaise qui vient de cloner l'ADNc codant pour le ligand de Fas, à partir d'une banque d'ADNc d'un hybridome cytotoxique, réexprimée dans les fibroblastes C.OS [2]. L'apparition du ligand de Fas sur les cellules transfectées est testée par fixation d'un récepteur soluble chimérique constitué des domaines extracellulaires de Fas fusionnés avec le fragment Fc des immunoglobulines. Il s'agit d'une glycoprotéine transmembranaire de type II formée de 278 acides aminés qui, lorsqu'elle est exprimée par les cellules COS, peut induire l'apoptose des cellules Fast. Le ligand de Fas, ainsi identifié, appartient à la famille du TNF et est exprimé principalement par les thymocytes, les splénocytes activés et dans les testicules. Comme le TNF, le ligand de Fas peut être libéré dans le milieu extracellulaire tout en gardant son activité biologique. Il n'est pas impossible que la libération excessive de la forme soluble du ligand de Fas puisse être pathogène chez l'homme.

[1. Kahn A, Briand P. médecine/sciences 1993; 9: 663-5.]

[2. Suda T, et al. Cell 1993; 75 : 1169-78.]

Le CD46 est le récepteur du virus de la rougeole. Le virus de la rougeole est responsable d'une maladie éruptive aiguë, grave par ses complications neurologiques possibles et responsable d'une immunodépression transitoire mais profonde. Par ses complications directes ou indirectes, la rougeole est une des premières causes de mortalité infantile dans les pays en voie de développement [1]. Une meilleure connaissance des mécanismes d'entrée du virus de la rougeole dans sa cellule cible est essentielle pour la compréhension de la biologie du virus. Le spectre d'hôte naturel du virus de la rougeole étant restreint à l'homme et à certaines espèces de singe, il a été postulé que ce virus utilise, pour son entrée dans la cellule, un récepteur spécifique de l'homme. Les travaux précédents avaient permis de caractériser la nature biochimique de ce récepteur et d'obtenir un anticorps monoclonal (MC 120.6) dirigé contre une glycoprotéine de $57 / 67 \mathrm{kDa}$. Cet anticorps inhibe la fixation, la fusion et l'infection des cellules humaines par le virus de la rougeole. Par immunopurification à l'aide de cet anticorps MC: 120.6 une protéine pure a été obtenue et un échantillon soumis à un microséquençage. La séquence obtenue est identique à la séquence $\mathrm{N}$ terminale du CD46 humain [2]. La fonction de CD46 est d'interrompre la cascade amplificatrice de l'activation du complément en inhibant l'activité des composants C4b et C.3b, et donc de protéger les cellules humaines de la lyse par le complément autologue. La transfection de cellules de souris, normalement non permissives au virus de la rougeole, avec un vecteur d'expression codant pour le CD46 humain a permis à l'équipe de C. Rabourdin-Combe (Lyon France) de montrer que l'expression du CD46 confère la capacité de fixation du virus de la rougeole et de formation de syncylia induite par les glycoprotéines $\mathrm{H}$ et $\mathrm{F}$ du virus. L'expression de CD46 dans des cellules M12 (lymphome B) de souris permet également le cycle réplicatif du virus et rend donc ces cellules permissives à l'infection par le virus de la rougeole. En outre, l'utilisation des transfectants M12CD46 permet le ciblage du virus, via la glycoprotéine d'enveloppe $\mathrm{H}$, sur la molécule CD46; dans ces conditions, les antigènes viraux $\mathrm{H}$ et NP sont très efficacement présentés aux lymphocytes $\mathrm{T}$ $\mathrm{CD}^{+}[3]$. L'obtention de souris transgéniques exprimant la molécule CD46, capables de répliquer le virus et donc de développer une maladie voisine de la rougeole, est le prochain objectif. Ce modèle animal devrait permettre de mieux comprendre la physiopathologie de l'infection rougeoleuse et, en particulier, d'identifier les mécanismes de l'immunosuppression survenant au moment de la phase éruptive. Il devrait également permettre de vérifier l'efficacité de nouveaux vaccins antirougeoleux.

[1. Lafaix C. médecine/sciences 1990 ; 6 (suppl. $\mathrm{n}^{\circ} 7$ ) : 12-8.]

[2. Naniche D, et al. J Virol 1993; 67 : 6025-32.]

[3. Gerlier D, et al. J Exp Med 1994 (sous presse).] 
Influence du polymorphisme sur la spécificité des transporteurs de peptides TAP. Le numéro de novembre de médecine/sciences a proposé à ses lecteurs une série d'articles concernant les mécanismes d'identification, par le système immunitaire, des cellules tumorales ou infectées $\left(\left(\mathrm{m} / \mathrm{s} n^{\circ} 11\right.\right.$, vol. 9 , p. 1284), [1, 2]). Les cellules anormales sont détectées car elles exposent à leur surface des molécules de classe I du complexe majeur d'histocompatibilité (CMHI) associées à des peptides antigéniques. Ces peptides sont le plus souvent produits dans le cytosol et s'associent au CMH-I dans le réticulum endoplasmique vers lequel ils sont "transloqués" par deux transporteurs (TAPl et 2). Même si l'importance de l'acide aminé en position C-terminale du peptide a été soulignée, les grandes lignes de la spécificité des TAP vis-à-vis des substrats peptidiques ne sont pas encore définies. Alors que chez l'homme et la souris le polymorphisme est très limité, des variations considérables de séquence existent entre les différents allèles chez le rat. Un travail récent vient de montrer, chez le rat, comment ce polymorphisme peut affecter la nature des peptides présentés aux lymphocytes T par le CMH-I [3]. En reconstituant in vitro le transport des peptides dans les microsomes, les auteurs remarquent qu'un allèle TAP2 (appelé cimb) a une préférence marquée pour les peptides ayant certains résidus hydrophobes à leur extrémité carboxylique alors qu'un autre allèle (cima est beaucoup moins sélectif. En fait, une fraction seulement des peptides transportés par cima est aussi transportée par cimb. Les expériences d'inhibition du transport d'un peptide par d'autres peptides sont en accord avec l'utilisation d'un site accepteur unique sur le transporteur. Cela n'élimine pas pour autant la possibilité que plusieurs sites de reconnaissance existent mais ne peuvent pas être utilisés simultanément. Dans ce cas précis des transporteurs de peptides chez le rat, le polymorphisme est bien un facteur limitant la diversité des peptides «transloqués » dans le réticulum endoplasmique. Le CMH-I lui-même, à cause de son polymorphisme, ne peut s'associer qu'aux peptides répondant à certaines exigences de taille et de charge. Par conséquent, la diversité des peptides capables de s'associer au CMH-I et d'être présentés aux lymphocytes $\mathrm{T}$ sera d'autant plus réduite que les critères imposés par TAP divergent de ceux imposés par le CMH-I. Il faut, cependant, relativiser l'importance de ce phénomène qui, s'il existe dans les lignées hautement sélectionnées de laboratoire, semble être exclu des autres populations par la sélection naturelle.

[1. Lotteau V. médecine/sciences $1993 ; 9$ : 1179-81.]

[2. Bahram S. médecine/sciences 1993; 9 : 1204-13.]

[3. Heemels MT, et al. Science 1993 ; 262 : 2059-63.]

Les mâles sont pressés. Depuis 1969, une biologiste londonienne, Ursula Mittwoch, soutient que les embryons mâles des mammifères, dès le stade deux cellules, se développent plus rapidement que les embryons femelles [1]. Selon cet auteur, il s'agit, pour les mâles, d'une véritable course contre la montre leur permettant de développer leurs organes sexuels et leur propre secrétion de testostérone avant de subir l'influence castratrice des oestrogènes sécrétés en quantité croissante par les ovaires maternels, puis le placenta. Lorsque la production de testostérone n'est pas assurée à temps, les gonades mâles soumises à l'influence des hormones maternelles et placentaires se développent en ovaires. Ces résultats semblent être confirmés dans différentes espèces, notamment la souris et les bovins. Selon U. Mittwoch, le prix à payer pour cette accélération du développement pourrait être la longévité réduite des mâles par rapport aux femelles. En d'autres termes, cette précipitation masculine pourrait perdurer, aboutissant à des individus au métabolisme basal plus important que chez les femelles, et, dans l'espèce humaine, vivant de cinq à huit ans de moins, en moyenne. Récemment, certains proposaient une variation sur ce thème du mâle pressé. Partant de l'observation que, en moyenne, les hommes dorment moins que les femmes, ils en extrapolaient que, en réalité, la vie éveillée était environ aussi longue dans les deux sexes! En d'autres termes, le drame des mâles serait qu'ils font tout trop vite...

[1. Vines G. The New Scientist 1993; $\left.140\left(n^{\circ} 1903\right): 18.\right]$

Daf-1, la cible de l'antagonisme entre les signaux relayés par les tyrosine kinases et l'AMP cyclique. Dans de nombreux systèmes, l'AMP cyclique et les agents qui en augmentent la concentration s'opposent à l'action de cytokines ou de facteurs de croissance dont l'effet passe par des tyrosine kinases et par les petites protéines $\mathrm{G}$ de la famille Ras. Par exemple, une lignée de fibroblastes de rat surexprimant le récepteur de l'EGF (epidermal growth factor) répond à ce facteur de croissance par une phosphorylation de la MAP kinase p42 [1]. Cette phosphorylation est bloquée par l'AMP cyclique, les stimulateurs de l'adénylyl cyclase et les inhibiteurs des phosphodiestérases. Le mécanisme de cet effet de l'AMP cyclique est la phosphorylation de la protéine $\mathrm{p} 75^{\text {raf }}$ sur la sérine 43. Une fois phosphorylée, la protéine Raf ne semble plus interagir avec la protéine Ras ce qui pourrait expliquer une interruption de la transmission du signal qui, passant de Ras à Raf, aboutit aux phosphorylations en cascade de la MAP kinase kinase et de la MAP kinase. Ces résultats sont un exemple supplémentaire de l'interconnexion, synergique ou antagoniste, des différentes voies de transmission intra-cellulaires des signaux. [1. Wu J, el al. Science 1993; 262 : 1065-69.] 